\title{
Alpha-mangostin attenuates oxidative stress and inflammation in adjuvant-induced arthritic rats
}

\author{
Yiwen Xu, Huimin Zhou and Lin Cai ${ }^{*}$ \\ Department of Orthopaedics, Zhongnan Hospital of Wuhan University, Wuhan, China
}

*For correspondence: Email: lincai137@hotmail.com; Tel: 0086-13476923997

Sent for review: 20 May 2017

Revised accepted: 12 October 2017

\begin{abstract}
Purpose: To examine the protective effect of $\alpha$-mangostin on Freund's adjuvant-induced rheumatoid arthritis (RA) in rats.

Methods: Male Wistar rats were divided into four groups $(n=6)$. The rats were made arthritic using a single intradermal injection of $0.1 \mathrm{ml}$ complete Freund's adjuvant. $\alpha$-Mangostin (100 mg/kg, i.p.) was administered for 21 days. After the experimental period, the animals were sacrificed and various biochemical markers were assessed.

Results: Arthritis-induced rats showed a significant $(p<0.05)$ increase in malondialdehyde $(16.28 \pm$ 1.43 vs $7.22 \pm 1.03$ ) and nitric oxide (3.82 \pm 0.87 vs $6.12 \pm 1.23$ ) with a concomitant reduction of antioxidants in the paw tissue. a-Mangostin intervention significantly $(p<0.05)$ reduced malondialdehyde and nitric oxide levels and restored antioxidant levels to normalcy by mitigating oxidative stress. The arthritic rats exhibited elevated tumor necrosis factor- $\alpha$ (TNF- $\alpha$ ) and interleukin- 6 (IL-6) serum concentrations, upregulated TNF- $\alpha$ and IL-6 protein levels, and upregulated nuclear factor $\kappa B(N F-\kappa B) m R N A$ expression in paw tissues. Treatment with $\alpha$-mangostin significantly $(p<0.05)$ reduced serum levels and downregulated inflammatory markers to normalcy.

Conclusion: The results suggest that $\alpha$-mangostin displayed a protective effect against adjuvantinduced arthritis in rats mediated through antioxidant and anti-inflammatory effects.
\end{abstract}

Keywords: Rheumatoid arthritis, Freund's adjuvant, $\alpha$-Mangostin, Oxidative stress, Inflammation

Tropical Journal of Pharmaceutical Research is indexed by Science Citation Index (SciSearch), Scopus, International Pharmaceutical Abstract, Chemical Abstracts, Embase, Index Copernicus, EBSCO, African Index Medicus, JournalSeek, Journal Citation Reports/Science Edition, Directory of Open Access Journals (DOAJ), African Journal Online, Bioline International, Open-J-Gate and Pharmacy Abstracts

\section{INTRODUCTION}

Rheumatoid arthritis (RA) is a chronic inflammatory clinical condition characterized by neutrophil infiltration in the synovium and synovial enlargement. This causes pannus formation, which then damages bones and cartilage leading to joint malformation [1]. RA affects approximately $1 \%$ of the global population. RA patients have a decreased quality of life due to extended therapy resulting in resistance, adverse toxic events, and high treatment costs [2]. Many factors are involved in RA etiology including genetic predisposition, viral and bacterial infections, altered immune status, impaired cognitive conditions, sedentary life style, and environmental hazards [3]. Reactive oxygen species (ROS) generation is also implicated in autoimmune diseases because of the association between oxidative stress and similar diseases [4].

Mounting evidence suggests that ROS, like superoxide and hydroxyl radicals, have a predominant role in RA etiology [5]. Furthermore, nitric oxide (NO) is a potent reactive nitrogen species that interacts with a superoxide radical to form a cytotoxic product, peroxynitrite, which 
eventually causes synovial damage [6]. Inflammation then induces joint disease and synovial damage. Inflammatory mediators, including tumor necrosis factor-alpha (TNF- $\alpha$ ) and interleukin-6 (IL-6), play a toxic role in RA [7]. In addition, a major inflammatory mediator, nuclear factor (NF)-KB, is associated with joint damage during $R A$.

Currently, anti-inflammatory drugs such as NSAIDs, disease-modifying anti-rheumatic drugs (DMARDs), steroid therapy, anti-TNF-a drugs, and monoclonal antibodies are the primary treatment options for RA [8]. However, usage of these drugs is restricted because of their toxic effects and high treatment cost. Another treatment option is to increase the body's antioxidant supply during free radical encounters via antioxidant intake through diet or as a supplement.

Alpha-mangostin ( $\alpha$-mangostin) is a therapeutic xanthone found in the Garcinia mangostana fruit pericarp. It has a high safety rating and is effective in the treatment of various pathological conditions [9]. Interestingly, earlier reports underscore the anti-inflammatory potential of $\alpha$ mangostin in an array of preclinical studies [10]. Based on the scientific evidence, a-mangostin may be an effective therapeutic agent in the amelioration of RA.

\section{EXPERIMENTAL}

Alpha-mangostin and complete Freund's adjuvant (CFA) were procured from SigmaAldrich (St. Louis, MO, USA).

\section{Animals}

Twenty four male Sprague - Dawley rats weighing 170- $200 \mathrm{~g}$, were maintained in temperature- and humidity-controlled animal houses under 12-h light/12-h dark cycle. The experimental animals were treated with standard diet pellets and water ad libitum. All experiments were carried as per the animal care protocol approved (ref no. AK3443111) by the Institutional Animal Ethics Committee (IAEC) of the Department of Orthopaedics, Zhongnan Hospital of Wuhan, Wuhan, China, and also complied with international guidelines [11].

\section{Study groups}

The animals were randomized into four different groups $(n=6)$ as follows: Group I (Control) received saline $(1.5 \mathrm{~mL} / \mathrm{kg}, \mathrm{p} .0)$ for 21 days: Group 2 (AIA) - CFA - induced arthritis (AIA) for 21 days; Group 3 (Man) - received a-mangostin alone (100mg/kg, p.o.) for 21 days; and Group 4 $(\mathrm{AIA}+)$ - received $\alpha$-mangostin (100 mg/kg; p.o) $1 \mathrm{hr}$ before complete Freund's adjuvant injection and continued for 21 days.

The animals were made arthritic by a single administration of complete Freund's adjuvant $(0.1 \mathrm{~mL} ; 10 \mathrm{mg} / \mathrm{mL})$ into the rat hind paw region via intra dermal route.

\section{Preparation of tissue homogenate}

At the end of the study, the blood was withdrawn and collected in heparinised BD vacutainer. Then the serum was separated by centrifugation at $2000 \times \mathrm{g}$ for $20 \mathrm{~min}$. The paw tissues were quickly removed, washed in ice-cold saline stored at $-80{ }^{\circ} \mathrm{C}$ until required.

\section{Evaluation of antioxidants}

SOD level and GSH content in paw tissue homogenate were estimated using commercially available kits (Nanjing, Jiancheng Co., China).The protein content was estimated using bovine serum albumin as a standard.

\section{Assessment of lipid peroxidation and nitrite}

Malondialdehyde (MDA) is a suitable marker for assessing lipid peroxidation and nitric oxide (NO) was measured using the kit supplied by (Nanjing Jiancheng Bioengineering Institute, Nanjing, Jiangsu Province, China).The protein content was estimated using a standard bovine serum albumin.

\section{Assay of cytokines}

Serum concentrations of IL- 6 and TNF- $\alpha$ were estimated by ELISA method with a commercial kit (ADL Co., San Diego, CA, USA).

\section{Western blot analysis of inflammatory proteins}

Extraction of paw tissue proteins was done using RIPA lysis buffer kit (Applygen Technologies Inc., China). Then the protein samples $(20 \mu \mathrm{g})$ were resolved by $10 \%$ sodiumdodecyl sulphate polyacrylamide gel electrophoresis (SDS-PAGE) Rabbit anti - IL - 6 and rabbit anti - TNF - a (1 : 400) were used as the primary standards. After washing, the blot was incubated with a secondary antibody, goat anti-mouse IgG conjugated to peroxidase (Santa Cruz, USA). The antigen- antibody reaction was estimated by ECL detection kit (Applygen Technologies Inc, China) and the protein bands were observed by Gel Doc XR system (Bio-Rad, USA). 


\section{RT-PCR analysis of NF-KB}

From the frozen paw tissue sample the RNA was isolated and purified using RNAase (Sigma, USA) as per manufacturer's instruction. The cDNAs was prepared using $1 \mu \mathrm{g}$ of the purified RNA sample. The oligonucleotide primers used in the study were as follows: NF- KB (P65)(381 bp):5' CCT ATC CAC GAC AAC CTT GC 3' (forward),5'CAT AGA TGC TGC TGA CCC AAC 3' (reverse); $\beta$ - actin (493 bp):5'-GTG GGGCGC CCA GGC ACC A-3' (forward) and 5'-GCT CGG CCG TGGTGG TGA AGC-3' (reverse).The reaction was carried out for 30 cycles as follows: 30s denaturing at $95{ }^{\circ} \mathrm{C}$ followed by a 30sannealing step at $57^{\circ} \mathrm{C}$. Finally the extension step was carried out for $1 \mathrm{~min}$ at $72{ }^{\circ} \mathrm{C}$. The protein band density value using the digital image system (Motic Images Advanced 3.2).

\section{Statistical analysis}

The data are presented as mean \pm SD (standard deviation). Differences between the groups were analyzed by one-way analysis of variance (ANOVA) followed by Tukey's test to assess the significance. $P<0.05$ was taken as statistically significant. SPSS software, version 13, was used for statistical analysis.

\section{RESULTS}

In the arthritis group, the malondialdehyde (MDA) level and NO was higher $(p<0.05)$ than in the control rats. $\alpha$-Mangostin treatment significantly reduced the MDA and NO level and thus inhibited the oxidative stress (Figure 1).

In the arthritis condition, the SOD, GPx, and GSH levels were significantly $(p<0.05)$ decreased in arthritic rats compared with the control rats. However, treatment with $\alpha$ mangostin significantly boosted the antioxidant levels to normalcy (Figure 2). In the present study, the serum concentration of IL-6 and TNF$\alpha$ were significantly $(p<0.05)$ elevated in the arthritis group. Treatment with $\alpha$-mangostin significantly $(p<0.05)$ decreased the cytokine levels mediated through its anti-inflammatory effect (Figure 3).
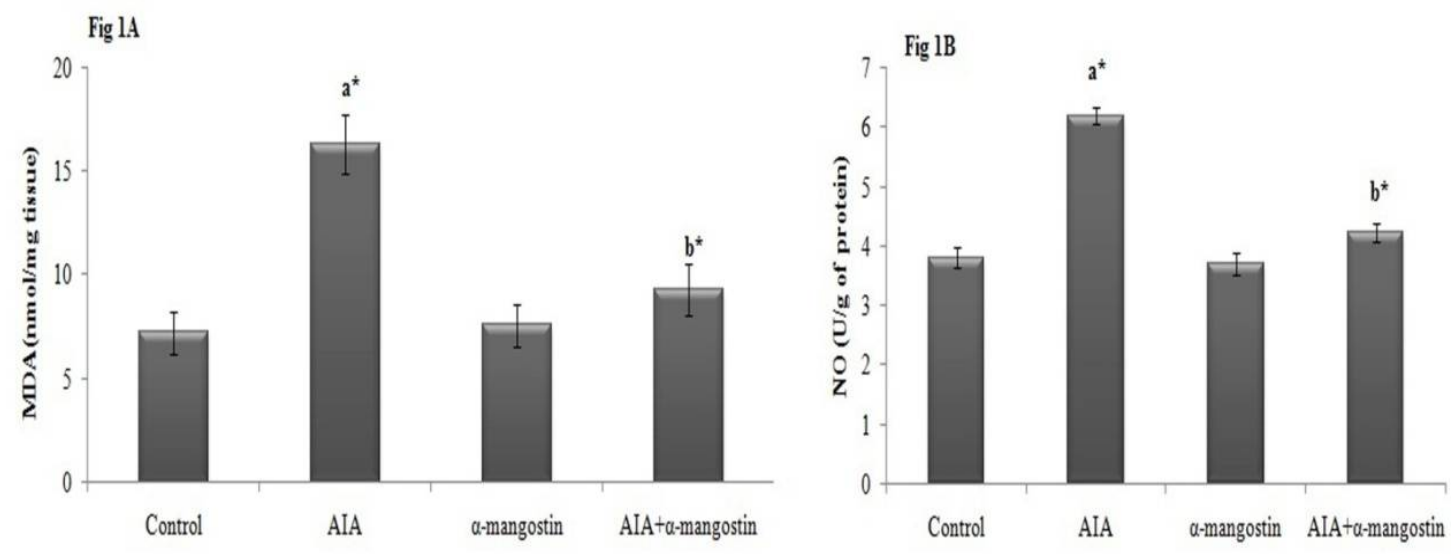

Figure 1: Effect of a-mangostin and AIA on MDA level (A) and NO (B) in paw tissue. Values are expressed as mean \pm S.D. for six rats in each group. Comparisons between control and (a) AIA; (b) AIA + $\alpha$-mangostin; *statistically significant $(p<0.05)$; AIA, adjuvant-induced arthritis
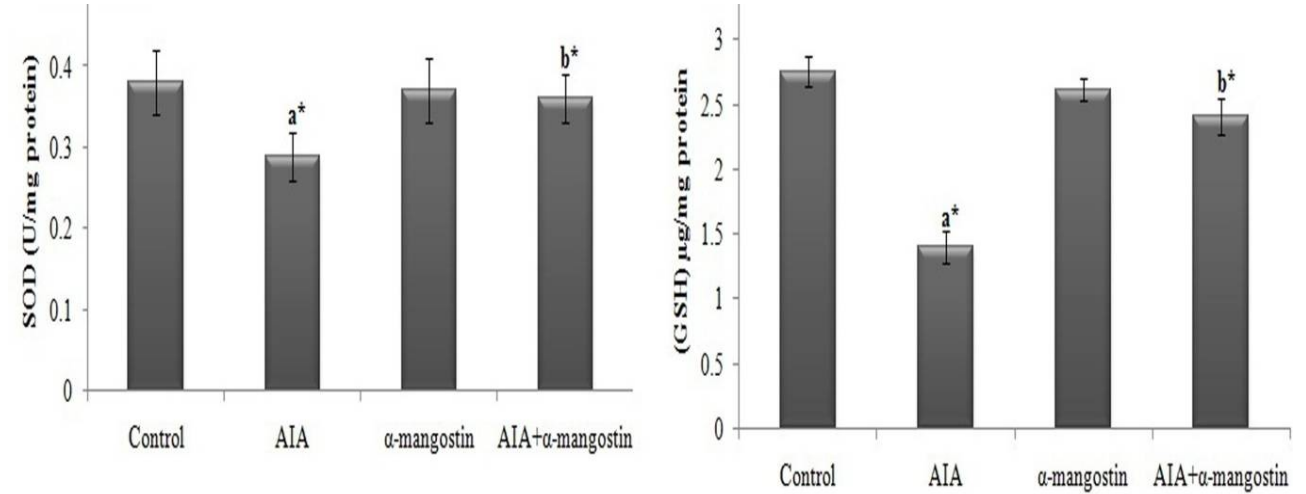

Figure 2: Effect of a-mangostin and AIA on antioxidant levels in paw tissue. (A) SOD (U/mg protein); (B) GSH $(\mu \mathrm{g} / \mathrm{mg}$ protein). Values are expressed as mean \pm S.D. for six rats in each group. Comparisons between control and (a) AIA; (b) AIA + a-mangostin; *statistically significant $(p<0.05)$; AIA, adjuvant-induced arthritis 

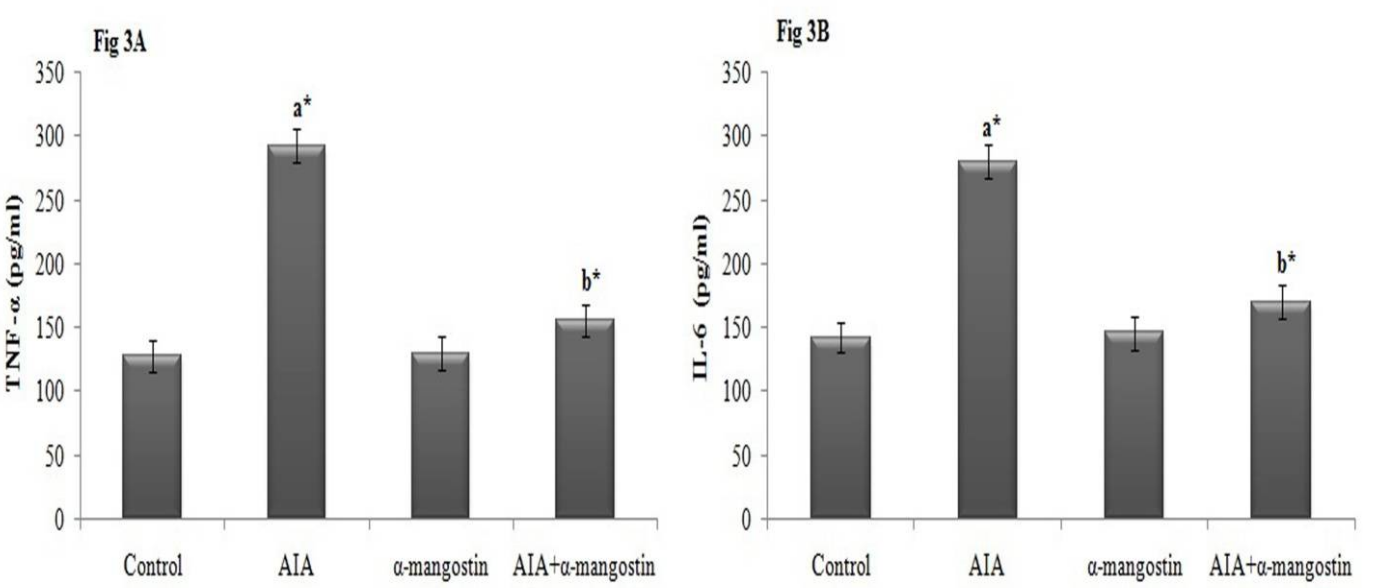

Figure 3: Pro-inflammatory cytokine levels in serum. (A) TNF- $\alpha(\mathrm{pg} / \mathrm{mL})$; (B) $\mathrm{IL}-6(\mathrm{pg} / \mathrm{mL})$. Values are expressed as mean \pm S.D. for six rats in each group. Comparisons between control and (a) AIA; (b) AIA + a-mangostin; *statistically significant $(p<0.05)$; AIA, adjuvant-induced arthritis
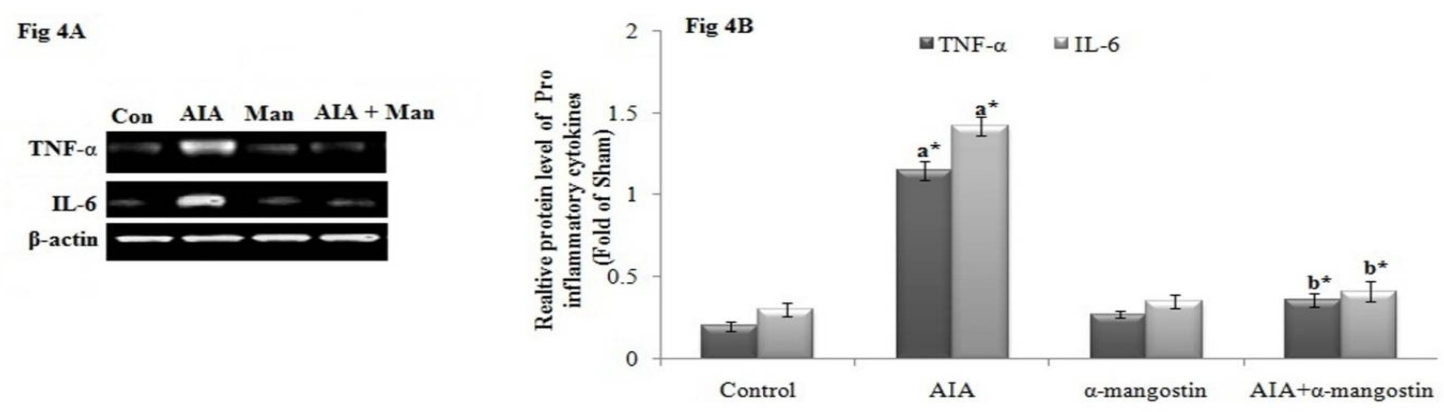

Figure 4: Western blot analysis of pro-inflammatory cytokines in paw tissues. (A) AIA rats displayed upregulated TNF- $\alpha$ and IL-6 expression whereas a-mangostin treatment downregulated the expression to normalcy. (B) Relative expression of pro-inflammatory cytokines (TNF- $\alpha$ and IL-6). Values are expressed as mean \pm SD $(n=6)$; comparisons between control and (a) AIA; (b) AlA + a-mangostin; *statistically significant $(p<0.05)$; AIA, adjuvant-induced arthritis

The arthritic rats displayed upregulated IL-6 and TNF- $\alpha$ protein levels in paw tissue. However, compared with the arthritis group, treatment with $\alpha$-mangostin attenuated the IL- 6 and TNF- $\alpha$ level in the paw tissue (Figure 4). The relative expression of IL- 6 and TNF- $\alpha$ were significantly $(p<0.05)$ higher in the arthritis group than the control. The arthritic rats treated with $\alpha$ mangostin showed a significant $(p<0.05)$ decrease in the protein level of the proinflammatory cytokines (Figure 4).

There was a significant increase in NF-KB expression in the paw tissue samples from arthritis-induced rats. However, therapeutic intervention using a-mangostin significantly reduced the levels NF-KB mRNA expression in the paw tissues (Figure $5 \mathrm{~A}$ ). The relative NF-KB mRNA expression level showed a significant $(p<$ $0.05)$ increase in the arthritis group. The arthritic rats treated with $\alpha$-mangostin showed downregulation of NF-kB mRNA expression (Figure 5B).

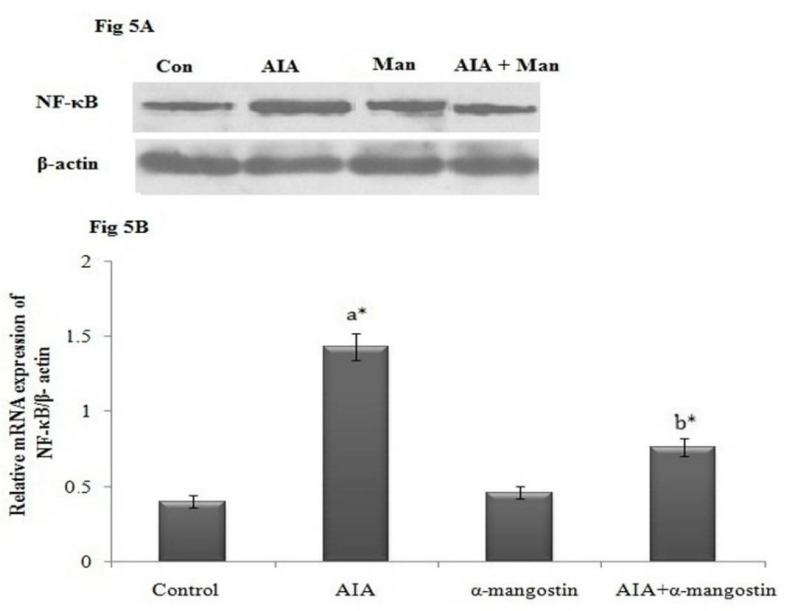

Figure 5: Effect of $\alpha$-mangostin and AIA on RT-PCR analysis of NF-KB mRNA expression in paw tissues. (A): In AIA rats, upregulated mRNA expression of NF$\mathrm{KB}$ was observed and a-mangostin treatment attenuated the inflammation by downregulating NF-kB expression. (B) Relative NF-kB mRNA expression. Values are expressed as mean $\pm S D(n=6)$; comparison between control and (a) AIA; (b) AIA + $\alpha-$ mangostin; *statistically significant $(p<0.05)$. AlA, adjuvant-induced arthritis 


\section{DISCUSSION}

Many preclinical reports substantiate that free radical stress has a prime role in the etiology of RA $[12,13]$. The generated ROS alters important cellular organs and forcefully damages the DNA,

MDA, a by-product from lipid peroxidation, attacks the lipid structures in cellular membranes and thus acts as reliable biological marker for free radical damage. MDA is a reactive molecule formed during the peroxidation of polyunsaturated fatty acids in the cell membrane [16]. In this study, an increased MDA level was observed in the paw tissue homogenate from arthritic rats, which is line with previous preclinical reports [17].

In this study, a-mangostin treatment significantly reduced the increased MDA level to normalcy. Phenolic and phenolic compounds exhibit their biological action by free radical scavenging [18] and inhibition of lipid peroxidative chains. The free radical scavenging potential of phenolic compounds is due to the presence of a hydroxyl and phenoxy functional group, which has the ability to donate the lone pair of electrons. The resultant phenolic radical converts to a quininemethide intermediate and is biotransformed via bile [19]. The effect of $\alpha$ mangostin on lipid peroxidation inhibition is due to its phenolic-like anti-oxidant property.

The human biological system encompasses a battery of antioxidant molecules to counter the free radical attack during RA [20]. The antioxidant defense mechanism against oxidative stress is initiated by SOD. During the oxidative stress pathway, the superoxide radical is released and the catalytic action of catalase converts GPx to $\mathrm{H}_{2} \mathrm{O}_{2}$ and molecular oxygen. Due to this reaction, vital biological organs and tissues are damaged from the decreased antioxidant levels. Finally, GSH counteracts the free radicals by quenching the single oxygen and hydroxide thereby protecting the tissues and organs [21]. Thus, in the present study, treatment with a-mangostin effectively reduced oxidative stress and significantly elevated the depleted level of both enzymatic and non-enzymatic antioxidants. Earlier work underscores the $\alpha$ mangostin efficacy on inflammatory conditions in a murine model in relation to oxidative stress [22].

TNF- $\alpha$ and IL- 6 are pro-inflammatory cytokines with a crucial role in the development of RA. Early phase elevated TNF- $\alpha$ and IL- 6 serum levels are the hallmark of RA [23]. In the present proteins, and lipids. During RA, the free radicals such as hydroxy radical, peroxynitrile, and hydrogen peroxide alter the lipid membrane structures and cause functional impairment of cellular components [14]. Lipid peroxidation is the key noxious event involved in RA [15].

investigation, treatment with $\alpha$-mangostin significantly downregulated TNF- $\alpha$ and IL-6 serum protein expression and upregulated paw tissue expression, which corroborates previous reports [24].

$\mathrm{NF}-\kappa \mathrm{B}$ is a key protein that mediates the inflammatory reaction during $\mathrm{RA}$. NF- $\kappa \mathrm{B}$ can induce pro-inflammatory cytokines, such as TNF$\alpha$ and IL-6, which can increase RA inflammation [25]. In this study, the elevated NF-kB mRNA level in arthritis-induced paw tissue was demonstrated. However, treatment with $\alpha$ mangostin down regulated NF-KB mRNA expression, and thus inhibited the generation of an inflammatory mediator leading to the alleviated pathological changes in RA [26].

\section{CONCLUSION}

a-Mangostin treatment significantly attenuates oxidative stress and inflammation in arthritic rats and is therefore potentially an alternative treatment for RA. However, further molecular studies are highly are required to elucidate the mechanism of action of a-mangostin.

\section{DECLARATIONS}

\section{Acknowledgement}

None provided.

\section{Conflict of Interest}

No conflict of interest associated with this work.

\section{Contribution of Authors}

The authors declare that this work was done by the authors named in this article and all liabilities pertaining to claims relating to the content of this article will be borne by them.

\section{Open Access}

This is an Open Access article that uses a funding model which does not charge readers or their institutions for access and distributed under the terms of the Creative Commons Attribution License (http://creativecommons.org/licenses/by/ 
4.0) and the Budapest Open Access Initiative (http://www.budapestopenaccessinitiative.org/rea d), which permit unrestricted use, distribution, and reproduction in any medium, provided the original work is properly credited.

\section{REFERENCES}

1. Ono $Y$, Inoue $M$, Mizukami $H$, Ogihara $Y$. Suppressive effect of Kanzo⿴囗口bushi冋to, a Kampo medicine, on collagentinduced arthritis. Biol Pharm Bull 2004; 27: 1406-1413.

2. Narendirakannan RT, Subramaniam S, Kandaswamy M. Anti-inflammatory and lysosomal stability actions of Cleome gynandra L. studied in adjuvant induced arthritic rats. Food Chem Toxicol 2007; 45: 1001-1012.

3. Kochi Y, Suzuki A, Yamada R, Yamamoto K. Genetics of rheumatoid arthritis: Underlying evidence of ethnic differences. J Autoimmun 2009; 32: 158-162.

4. McInnes IB, Schett G. The pathogenesis of rheumatoid arthritis. N Engl J Med 2011; 365: 2205-2219

5. Jaswal S, Mehta HC, Sood AK, Kaur J. Antioxidant status in rheumatoid arthritis and role of antioxidant therapy. Clin Chim Acta 2003; 338: 123-129.

6. Bauerova K, Bezek $S$ Role of Reactive Oxygen and Nitrogen Species in Etiopathogenesis of Rheumatoid Arthritis. Gen Physiol Biophys 1999; 18: 15-20.

7. Jazayeri JA, Carroll GJ, Vernallis AB. Interleuking6 subfamily cytokines and rheumatoid arthritis: Role of antagonists. Int Immunopharmacol 2010; 10: 1-8.

8. Colmegna I, Ohata BR, Menard HA. Current understanding of rheumatoid arthritis therapy. Clin Pharmacol Ther 2012; 91: 607-620.

9. Wang $\mathrm{MH}$, Zhang $\mathrm{KJ}$, Gu QL, Bi XL, Wang JX. Pharmacology of mangostins and their derivatives: $A$ comprehensive review. Chin J Nat Med 2017; 15: 81-93.

10. Guide for the care and use of laboratory animals, 8th Edition, Institute for Laboratory Animal Research, Division on Earth and Life Studies, The National Academic Press, Washington $D C$.

11. Jang HY, Kwon OK, Oh SR, Lee HK, Ahn KS, Chin YW. Mangostinxanthones mitigate ovalbumin-induced airway inflammation in a mouse model of asthma. Food Chem Toxicol 2012; 50: 4042-4050

12. Phillips DC, Dias HK, Kitas GD, Griffiths HR. Aberrant reactive oxygen and nitrogen species generation in rheumatoid arthritis (RA): causes and con-sequences for immune function, cell survival, and therapeutic intervention. Antioxid Redox Signal 2010; 12: 743-785.

13. Zheng L, Yang L, Wang Z, Chen C, Su Y. Protective effect of Esculin in adjuvant-induced arthritic (AIA) rats via attenuating pro-inflammatory cytokines and oxidative stress. Cell Mol Biol (Noisy-le-grand) 2015; 61: 1-5.

14. Hitchon CA, El-Gabalawy HS. Oxidation in rheumatoid arthritis. Arthritis Res Ther 2004; 6: 265-278.

15. Łuczaj W, Gindzienska-Sieskiewicz E, JarockaKarpowicz I, Andrisic L, Sierakowski S, Zarkovic N et al. The onset of lipid peroxidation in rheumatoid arthritis: consequences and monitoring. Free Radic Res 2016; 50: 304-313.

16. Pohanka M, Vobornikova I, Fusek J. Freund's complete adjuvant effect on BALB/C mice: an insight into inflammation and oxidative stress after immunity challenge. Bratis/ Lek Listy 2016; 117: 268-271.

17. Vysakh A, Ratheesh M, Rajmohanan TP, Pramod C, Premlal S, GirishKumar $B$ et al. Polyphenolics isolated from virgin coconut oil inhibits adjuvant induced arthritis in rats through antioxidant and anti-inflammatory action. Int Immunopharmacol 2014; 20: 124-130.

18. Gil MI, Francisco A, Barberan T, Pierce BH, Holcroft DM, Kader AA. Antioxidant activity of pomegranate juice and its relationship with phenolic composition and processing. J Agric Food Chem 2000; 48: 4581-4589.

19. Pan GX, Spencer L, Leary GJ. Reactivity of ferulic acid and its derivative towards hydrogen peroxide and peracetic acid. J Agric Food Chem 1999; 47: 3325.

20. Campo GM, Avenoso A, Campo S, Ferlazzo AM, AltavillaD, Calatroni A. Efficacy of treatment with glycosaminoglycans on experimental collagen-induced arthritis in rats. Arthritis Res Ther 2003; 5: R122-131.

21. Sindhu G, Ratheesh M, Shyni GL, Nambisan B, Helen A. Anti-inflammatory and antioxidative effects of mucilage of Trigonellafoenumgraecum (Fenugreek) on adjuvant induced arthritic rats. Int Immunopharmacol 2012; 12: 205-211.

22. Sampath $P D$, Kannan V. Mitigation of mitochondrial dysfunction and regulation of eNOS expression during experimental myocardial necrosis by alpha-mangostin, a xanthonic derivative from Garcinia mangostana. Drug ChemToxicol 2009; 32: 344-352.

23. Tanida S, Yoshitomi H, Nishitani K, Ishikawa M, Kitaori T, Ito $\mathrm{H}$ et al. CCL20 produced in the cytokine network of rheumatoid arthritis recruits CCR6+ mononuclear cells and enhances the production of IL-6. Cytokine 2009; 47: 112-118.

24. Sampath PD, Vijayaragavan $K$. Ameliorative prospective of alpha-mangostin, a xanthone derivative from Garcinia mangostana against beta-adrenergic catecholamineinduced myocardial toxicity and anomalous cardiac TNF-alpha and COX-2 expressions in rats. Exp Toxicol Pathol 2008; 60: 357-364.

25. Hu Z, Wang W, Ling J, Jiang C. a-Mangostin Inhibits $\alpha$ Synuclein-Induced Microglial Neuroinflammation and Neurotoxicity. Cell Mol Neurobiol 2016; 36: 811-820. 